\title{
On the Nature of the Anti-Tail of Comet Kohoutek (1973f). II. Comparison of the Working Model with Ground-Based Photographic Observations
}

\author{
ZDENEK SEKANINA \\ Center for Astrophysics, \\ Harvard College Observatory and Smithsonian Astrophysical Observatory, \\ Cambridge, Massachusetts 02138
}

AND

FREEMAN D. MILLER

Department of Astronomy, University of Michigan, Ann Arbor, Michigan 48104

Received May 13, 1975; revised June 3, 1975

\begin{abstract}
Photographic observations of the anti-tail of Comet Kohoutek (1973f), obtained at the Cerro Tololo Inter-American Observatory, are photometrically reduced and the results compared with a recently formulated working model of the antitail. The applied technique of photometric reduction is described, and the radial and transverse profiles of the anti-tail, corrected for the effects of atmospheric extinction and the variable intensity of the ambient sky, are derived. Brightness variations in time are also studied. The most important result reached so far is a quantitative confirmation of the previously suggested hypothesis, arguing that dust particles in the anti-tail suffered a significant loss in radius due to evaporation near the perihelion passage. We find that only particles initially larger than $100-150 \mu \mathrm{m}$ in diameter (at an assumed density of $1 \mathrm{~g} \mathrm{~cm}^{-3}$ ) survived. Numerically, however, this result is still preliminary, because the dynamical effect associated with particle evaporation remains to be explained. It is also tentatively suggested that the emission rate of the dust from the comet was probably comparable with the rates derived earlier for Comets Arend-Roland (I957 III) and Bennett (1970 II).
\end{abstract}

\section{INTRODUCTION}

A working model was recently formulated for the anti-tail of Comet Kohoutek (Sekanina, 1974; referred to hereafter as Paper I). The model was based on the Finson-Probstein (1968) theory of dust comets and fitted reasonably well the semiquantitative descriptions of the anti-tail by a number of observers, including the Skylab III astronauts. In the present paper, the radial and transverse brightness profiles and the time variations in the surface brightness of the anti-tail are established from plates of the comet taken at the Cerro Tololo Inter-American Observatory, near La Serena, Chile, and the working model is

Copyright 1976 by Academic Press, Inc. All rights of reproduction in any form reserved. Printed in Great Britain then tested quantitatively. The photographic material studied here was secured under the supervision of the second author. The photometric reduction and physical interpretation are due to the first author.

\section{The Michigan Program of Photographic Opservations of Comets}

The plates described in Section III form part of a program of comet observations initiated by the second author in 1950 with the Curtis-Schmidt telescope $(61 / 91 \mathrm{~cm}$, $\mathrm{f} / 3.5,96.6 \mathrm{~mm}^{-1}$ ) of the University of Michigan. In addition to unfiltered "blue" plates, exposures have been taken through red filters to suppress the $\mathrm{CO}^{+}$emissions of 
Type I tails relative to the scattered sunlight of the Type II tails, on which the former are often superposed. The extent to which the suppression is successful has varied from one comet to another (Miller, $1958,1962)$. The development of a model of dust tails by Finson and Probstein (1968) has stimulated interest in the study of the Michigan material, and analyses for Comets Seki-Lines 1962 III (Jambor, 1973) and Bennett 1970 II (Sekanina and Miller, 1973) have already appeared.

From the first, the comet-program plates have been photometrically calibrated with a 14-tube spot sensitometer designed by E. B. Weston; when the telescope was transferred from Michigan to Cerro Tololo, the sensitometer accompanied it. Since the precision of the sensitometer calibration is critical, the photometric scale was measured photoelectrically by W. Liller in 1951 and 1955 and by S. M. Simkin in 1965 . The average deviation from the mean scale of the three independent measurements for each spot is $\pm 0.04 \mathrm{mag}$; a more detailed discussion of the calibration tests can be found in a paper by Miller (1967). A calibration exposure is made for each comet photograph through the same filter used in the comet exposure and on a plate taken from the same box and developed with the comet plate. The length of the calibration exposure is the same as that for the comet.

\section{The Cerro Tololo Photographs}

A total of 12 calibrated plates were taken at Cerro Tololo with the Curtis-Schmidt telescope between January 16 and February 15, 1974. Five plates, taken on January 23, 24, and 26 and February 12 and 15, are 098-02 panchromatic emulsions combined with an RG1 filter to provide maximum sensitivity near $66000 \AA$ and to cut off the visible spectrum below $\lambda \approx 5900 \AA$. The photometric system thus obtained is similar to the $R$ system of Johnson and Mitchell (1962). The other seven plates, taken on January $16,17,20$, $21,23,25$, and 27 , are standard 103a-0 emulsions without filter. The red-sensitive plates are of primary interest, both because they span a longer interval of time and because they show the anti-tail better. The guiding of the January 24 plate was somewhat imperfect, so only the other four plates, two in January and two in February, have been photometrically studied. The four observations are listed in Table 1.

Of much concern has been the very low altitude of the comet. At the end of the exposure, the comet was only slightly over $10^{\circ}$ above the horizon on plates 15520 , 15687 , and 15703 and about $7^{\circ}$ above the horizon on 15554 ! Thanks to the excellent. sky at Cerro Tololo, the plates are still photometrically valuable, but the very low altitude of the comet is a source of two potential complications for the photometry of the plates, namely the effects of differential refraction and strongly variable sky brightness. In addition, atmospheric extinction, which is severe at these altitudes even in the red section of the spectrum, also needs to be accounted for.

TABLE I

('erro Tololo ReD-Sensitive Photographs of Comet Kohot'tek (098-0)2 EMulsion With an RGI Filter)

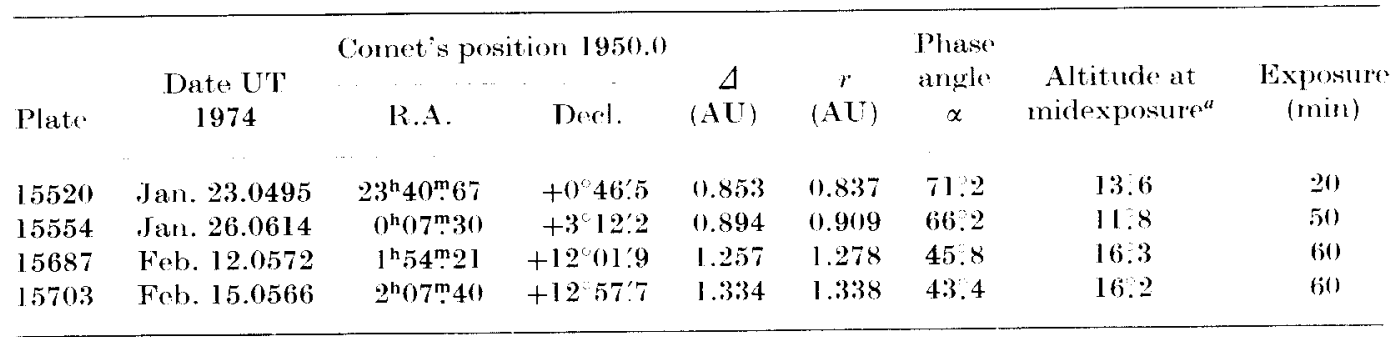

a Not corrected for a depressed horizon. 
The differential-refraction effect has been quantitatively analyzed following the procedure described in Appendix A. Numerically, refraction has been found to affect the angular distance from the nucleus on the four plates by less than $1 \%$, and the position angle, by no more than $0.1^{\circ}$ at an angular distance of $1^{\circ}$ from the nucleus and no more than $0.2^{\circ}$ at $2^{\circ}$.

The effect of variable sky brightness proved considerably more severe. It so happened that the brightest section of the anti-tail was directed almost exactly toward the horizon on all the plates, so that the maximum brightness gradient of the sky projected unforeshortened along the anti-tail. In addition, all exposures began during astronomical twilight (with the Sun's depression between $15^{\circ}$ and $17^{\circ}$ ), and almost the whole 15520 exposure was taken during twilight (with the Sun's depression at midexposure $17^{\circ}$ ). To demonstrate the extent of interference, we mention that the relative gradient of the sky brightness on plates 15520 nad 15554 reached a maximum rate of $7 \%$ per degree of altitude, while at the same time the intrinsic surface brightness of the anti-tail amounted to only some $4-6 \%$ of the sky brightness at $15^{\prime}$ from the nucleus and about $1-2 \%$ at $25^{\prime}$.

\section{The Technique of Photometric Reduction}

The technique for reducing the plates was essentially dictated by the troublesome observing conditions. Because of the complicated character of the sky-brightness variations near the horizon, it was virtually impossible to use the standard two-dimensional scans to map the sky "noise" over the area occupied by the anti-tail (see Fig. 1). Preferable, because more revealing, are one-dimensional radial tracings, passing through the comet's nucleus and effectively covering the whole anti-tail. Each radial scan is defined by the position angle, and the "noise" variations in the respective section of the antitail can be reasonably interpolated from the known field brightness outside the anti-tail (see Fig. 2). Nearby stars of

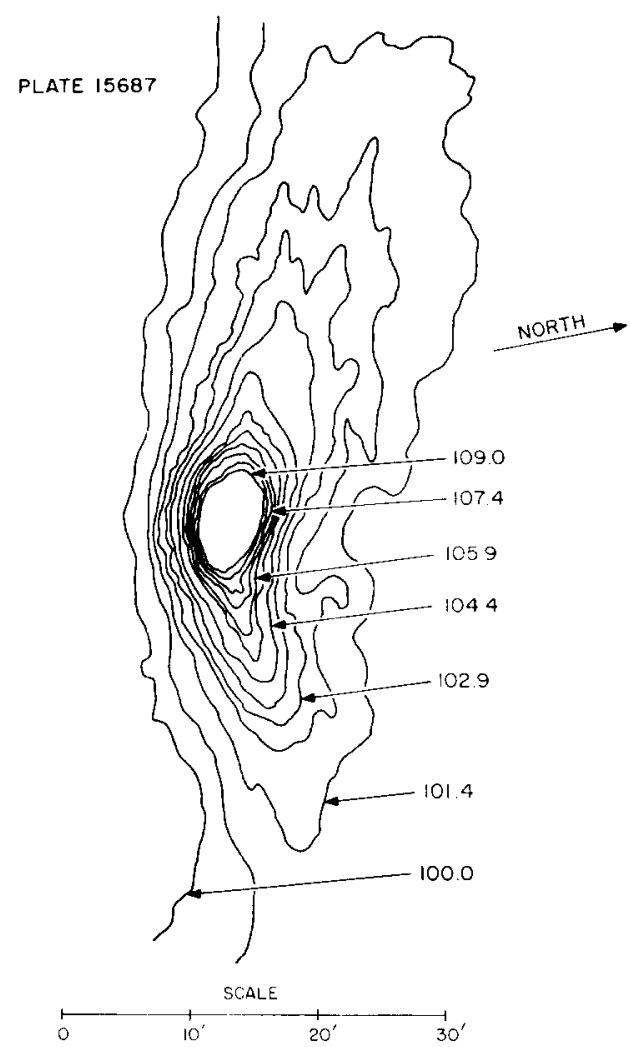

FIG. 1. A two-dimensional scan of plate 15687 . Scanning aperture is 9.7 by 9.7 . The anti-tail points to the bottom of the scan, and the regular dust tail to the top. The numbers indicate the relative surface brightness, uncorrected for the contribution from the sky.

known positions have been used to fix the position angles. The position of the comet on the plates was measured relative to the ambient stars and reduced routinely with the use of the method of dependences. The result has also been checked by computing the expected topocentric position of the comet from Marsden's (1974) definitive orbit. The measured and computed positions agree within $2^{\prime \prime}$ on plate 15520 , $7 "$ on $15554,8^{\prime \prime}$ on 15687 , and $13^{\prime \prime}$ on 15703 . These residuals can be accounted for entirely by the uncertainty in the location on the plates of the nucleus in the strongly overexposed coma, which is almost $2^{\prime}$ in diameter. The diffuse image of the comet's head is also responsible for an uncertainty in the distance scale of the scans, amounting to several percent at $10^{\prime}$ from the 


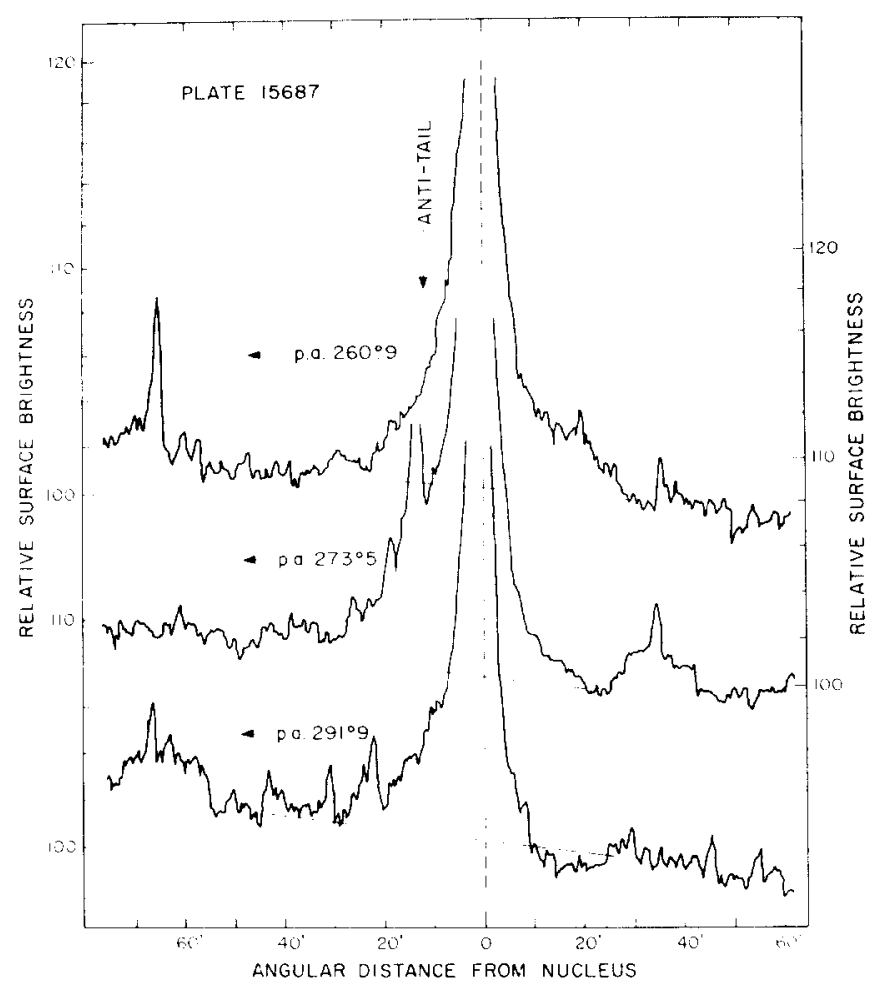

Fig. 2. Three radial scans of plate 15687 . Scanning aperture is 5"8 by 5"8. Note the variable sky brightness (dashed curves).

nucleus and dropping to somewhat more than $1 \%$ at $30^{\prime}$. Together with the images of the orientation-fixing stars, which appear as trails a few minutes of arc in length on the four plates, the comet's image is also responsible for an error in the position angle of the scans, which is estimated to be no more than about $\pm 0.5^{\circ}$. Recalling the effects introduced by differential refraction (Section III), we conclude that those effects are much smaller than the measurement errors and can therefore be safely neglected.

An important parameter of any photometric procedure, from a practical point of view, is the size of the scanning diaphragm. Its choice is governed by a compromise between the requirement of a sufficiently high resolving power on the one hand and that of a smooth image reasonably free from the effects of emulsion granulation on the other. After consulting Miller's (1967) rule of thumb and some experimenting, the diaphragm used in the radial scans was fixed at $60 \mu \mathrm{m}$ by $60 \mu \mathrm{m}$, i.e., $5 " 8$ by $5 " .8$. With this diaphragm size, and after accounting for the effects of interfering stars and plate flaws, we could read the surface brightness of the anti-tail from the radial scans (such as the ones in Fig. 2) with a precision of a few tenths of $1 \%$ of the sky brightness. 'Thus, near the direction of peak intensity the error in the relative. surface-brightness data of the anti-tail is estimated at about $\pm 10 \%$ at $15^{\prime}$ from the nucleus and at some $\pm 25 \%$ at $25^{\prime}$.

The photoelectrically calibrated spots were measured with a Macbeth densitometer to obtain the characteristic curves of the plates, and the photographic density was then converted to relative intensities.

\section{Absolute Calibration of the Plates}

To calibrate the surface brightness of the anti-tail in absolute units, two or three stars have been selected on each photo- 
graph. Because of very dense cores of star trails on Curtis-Schmidt plates, only very faint stars, of $R$ magnitude 13 to 14 , could be chosen for calibration.

Strictly, each calibration star should have been scanned along as well as across its trail. However, inspection of the magnified trails of the calibration stars in a measuring machine has shown that virtually nothing can be gained by scanning the stars along their trails rather than by simply measuring their lengths, once the cross scans are available.

To get a meaningful profile of the calibration stars in the direction perpendicular to the trail, a slit diaphragm was used in the cross scans. The width of the slit in the scanning direction was fixed at $10 \mu \mathrm{m}$ (or just below $1^{\prime \prime}$ ), a size significantly smaller than the apparent width of the trails of the calibration stars (typically $50 \mu \mathrm{m})$. To avoid the effect of emulsion granulation, the length of the diaphragm along the trail was kept at $200 \mu \mathrm{m}$ (or about 19 "3), which represents about $10 \%$ of an average trail length on the plates. The measured length of the star trails depends on the star brightness and is also somewhat affected by differential refraction. An example of a scan of a calibration star is shown in Fig. 3, and the photometric reduction of these scans is described in Appendix B.
The coordinates of the calibration stars, none of which is listed in the SAO Star Catalog, have been determined by the method of dependences (using up to 10 ambient reference stars) and should be good to within $1^{\prime \prime}$ in each coordinate, except perhaps for the stars on plate 15554, where the accuracy may be lower.

The $R$ magnitudes of the calibration stars were kindly supplied to us by W. Liller. In brief, his procedure was based on establishing a relation between the $R$ magnitudes and the measured diameters of stars on the red prints of the Palomar Sky Survey. To determine the constants of this calibration relation, Liller used a number of stars from the Pleiades, whose $V$ magnitudes and $B-V$ indices (Iriarte, 1967) were converted to the $R$ magnitudes with the help of a $B-V$ versus $V-R$ relation fitted to the bright stars of known multicolor magnitudes (Iriarte et al., 1965). The absolute uncertainty of this calibration is estimated by Liller to be about $\pm 0.15 \mathrm{mag}$. Table II lists the calibration stars, whose magnitudes refer to outside the Earth's atmosphere.

An extinction rate of $0.07 \mathrm{mag}$ per air mass, also communicated to us by Liller, is applied to the measured $R$ magnitudes of the anti-tail in order to correct them for the atmospheric absorption at Cerro Tololo.

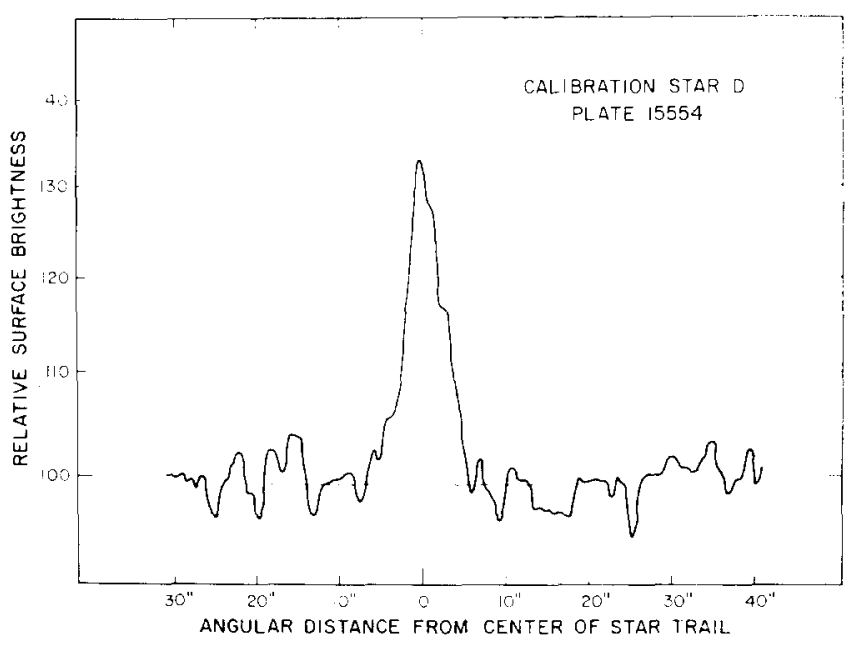

FIG. 3. An example of a scan across a calibration star's trail. Scanning slit is 1 ".0 by $19^{\prime \prime} 3$; the long side of the slit is parallel to the trail. The dashed line is the adopted sky brightness. 
TABLE II

Galibration Stars

\begin{tabular}{|c|c|c|c|c|}
\hline \multirow{2}{*}{$\begin{array}{c}\text { Calibration } \\
\text { star }\end{array}$} & \multirow[b]{2}{*}{ Plate } & \multicolumn{2}{|c|}{ Star's position 1950.0 } & \multirow{2}{*}{$\begin{array}{c}\text { Apparent } \\
R \text { magnitude } \\
\text { outside Earth's } \\
\text { atmosphere }\end{array}$} \\
\hline & & R.A. & Decl. & \\
\hline A & 15520 & $23^{\mathrm{h}} 42^{\mathrm{m}} 17^{\mathrm{s}} \cdot 90$ & $+0^{\circ} 40^{\prime} 46^{\prime \prime} 8$ & 13.34 \\
\hline B & 15520 & $23^{\mathrm{h}} 44^{\mathrm{m}} 37^{\mathrm{s}}: 16$ & $+0^{\circ} 48^{\prime} 10^{\prime \prime} 7$ & 13.04 \\
\hline (! & 15554 & $0^{\mathrm{h}_{0}} 05^{\mathrm{m}} 533^{\mathrm{s}} \cdot 42$ & $+318^{\prime} 17^{\prime \prime 2}$ & 12.95 \\
\hline D) & 15554 & $0^{\mathrm{h}} 06^{\mathrm{m}} 50^{\mathrm{s}} .05$ & $+3^{\circ} 22^{\prime} 55^{\prime \prime} 5$ & 13.49 \\
\hline $\mathrm{E}$ & 15687 & $1^{\mathrm{h}} 53^{\mathrm{m}} 300^{\mathrm{s}} \times 1$ & $+12^{\circ} 13^{\prime} 10^{\prime \prime} 9$ & 13.29 \\
\hline F & 15687 & $1^{\mathrm{h}} 55^{\mathrm{m}} 07883$ & $+12^{\prime} 03^{\prime} 40^{\prime \prime} 8$ & 13.12 \\
\hline $\mathrm{G}$ & 15703 & $2^{\mathrm{h}} 06^{\mathrm{m}} 011^{\mathrm{s}} 57$ & $+12^{\circ} 50^{\prime} 32^{\prime \prime} .7$ & 13.93 \\
\hline $\mathrm{H}$ & 15703 & $2^{\mathrm{h}} 06^{\mathrm{m}} 01^{\mathrm{s}} .88$ & $+12^{\circ} 50^{\prime} 48^{\prime \prime} 6$ & 14.54 \\
\hline J & 15703 & $2^{\mathrm{h}} 08^{\mathrm{m}} 07^{\mathrm{s}}: 86$ & $+13^{\circ} 23^{\prime} 45^{\prime \prime} 5$ & 13.69 \\
\hline
\end{tabular}

\section{The Results}

The photometric procedure described in the previous sections has been applied to the four plates listed in Table I. The absolute units used for the surface brightness are $S_{10}(\mathrm{red})$, the equivalent number of stars per square degree of the apparent $R$ magnitude 10. Figures 4-7 show the distribution of light in the anti-tail on the four nights as a function of position angle (transverse profiles). A comparison with the expected profiles predicted from the working model shows a certain degree of similarity. However, the anti-tail appears -primarily near the nucleus-to be broader than anticipated, perhaps because of the effect of nonzero ejection velocities of the dust particles, which was not taken into account in the formulation of the model. Also, Fig. 4 suggests a rather strong dependence of the position angle of the maximum brightness on the distance from the nucleus. On the other hand, the observed positions of the brightness maxima on the two February plates agree with the model within a few degrees, and the observed profiles at large distances from the nucleus (about $30^{\prime}$ ) on plate 15687 are fitted by the calculated profiles fairly well indeed.

The variations in the maximum surface brightness of the anti-tail with angular distance from the nucleus are displayed in Fig. 8. The slope of the radial profiles on the four nights agrees with the predicted

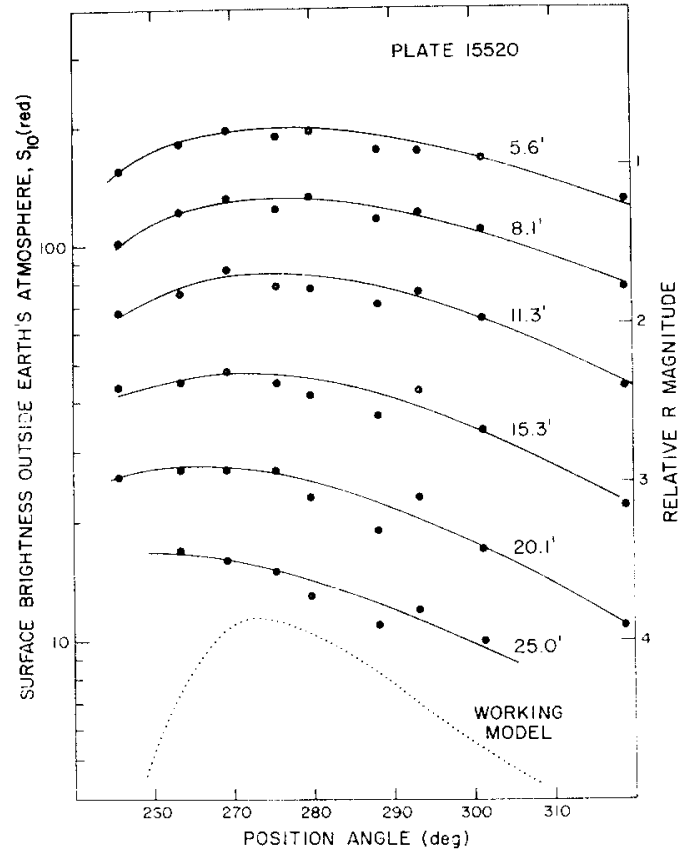

Fuc. 4. The distribution, in position angle, of surface brightness in the anti-tail of comet Kohoutek on plate 15520 at soveral angular distances from the nucleus. The surface bright. ness, expressed in $S_{10}$ units (tho equivalent number of magnitude 10 stars per square degree), refers to a color system similar to that of .Johnson's $I$ magnitudes and is corrected for atmospheric absorption (air mass $=0$ ). For the sake of comparison, the profile predicted by the working model is shown, in relative units, at the bottom. On the logarithmic scale, the predicted profile is virtually independent of the distane from the mucleus. 


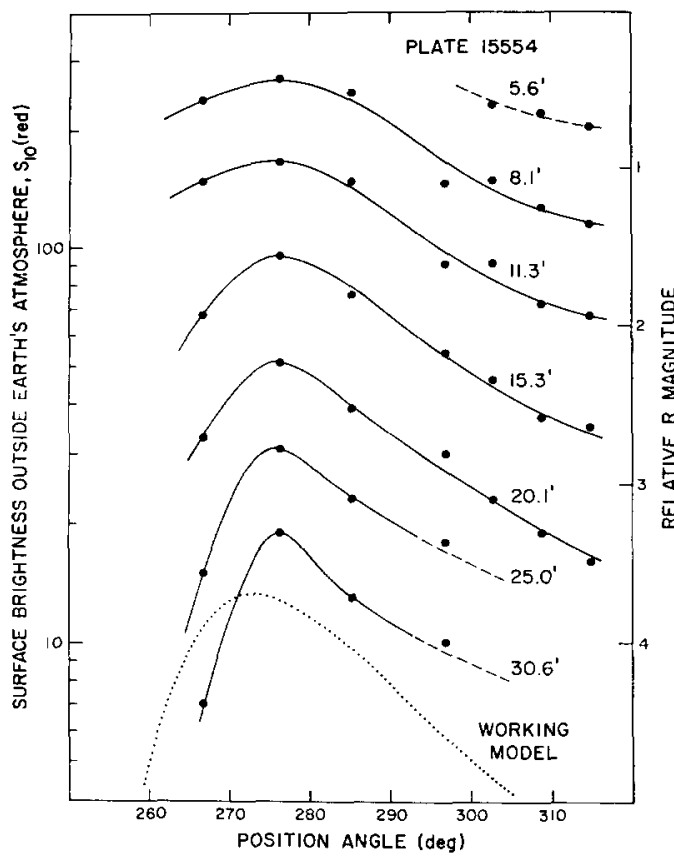

FIG. 5. The distribution, in position angle, of surface brightness in the anti-tail of Comet Kohoutek on plate 15554 at several angular distances from the nucleus. For details, see the caption to Fig. 4.

inverse 2.8 power law at distances from the nucleus exceeding $20^{\prime}$, but the surface brightness drops more slowly at smaller distances. In the next section, we submit a tentative interpretation of this effect in terms of a variable population index $s$ of the differential particle mass distribution $m^{-s} d m$ in the mass range under consideration, a case not considered in Paper I.

Finally, Fig. 9 is a plot of the maximum surface brightness of the anti-tail as a function of time and distance from the nucleus. The total error is estimated at $\pm 20 \%$ to $\pm 30 \%$ at $15^{\prime}$ from the nucleus and at probably more than $\pm 50 \%$ at $30^{\prime}$. Unfortunately, the 17-day gap between the second and third observations-the period of interference from the Moonmakes the actual curve of time variations rather indeterminate, and the fits shown in the figure are just one of many possibilities. However, we can be reasonably certain that the brightness was increasing fairly rapidly in late January, must have

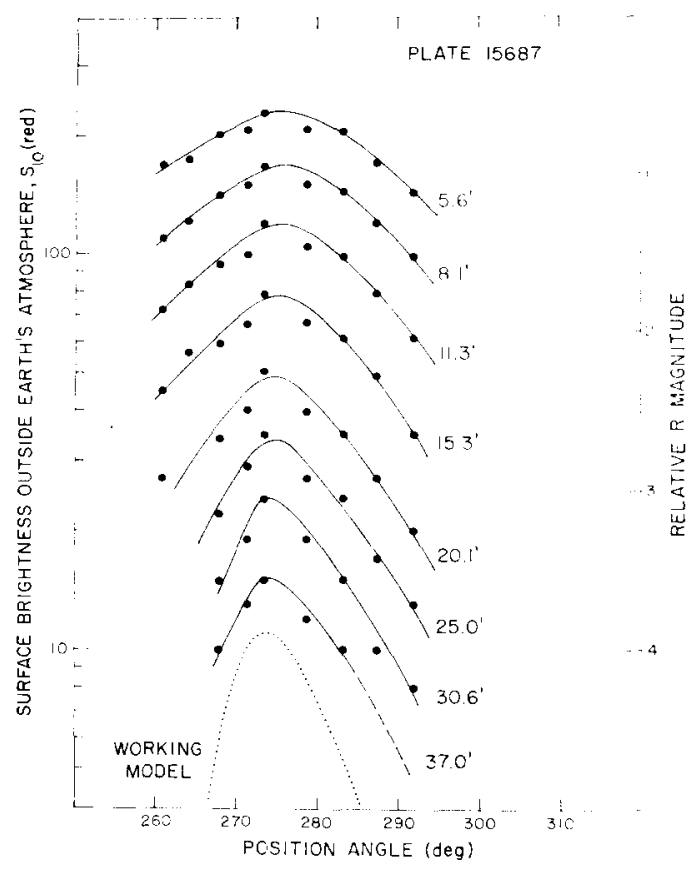

FIG. 6. The distribution, in position angle, of surface brightness in the anti-tail of Comet Kohoutek on plate 15687 at several angular distances from the nucleus. For details, see the caption to Fig. 4.

reached a maximum sometime around February 1, and was almost constant or somewhat subsiding in mid-February. Comparison of the observations with the working model shows some signs of resemblance to the predicted curves. The fit is better for the asteroidal-type phase law in late January and for a constant phase effect in mid-February.

Because of the differences between the observations and the working model in general and the variability of the radial gradient in particular, the relation between the surface-brightness distribution and the mass-loss rate of the dust from the comet is more complicated than suggested in Paper I and will have to be reassessed by means of an improved model. We note, however, that a cursory comparison of the Cerro Tololo plates with the Lowell Observatory prints (for the latter, see Paper I) suggests that the surface-brightness ratio of the anti-tail to the sky is likely to be lower than was adopted in 


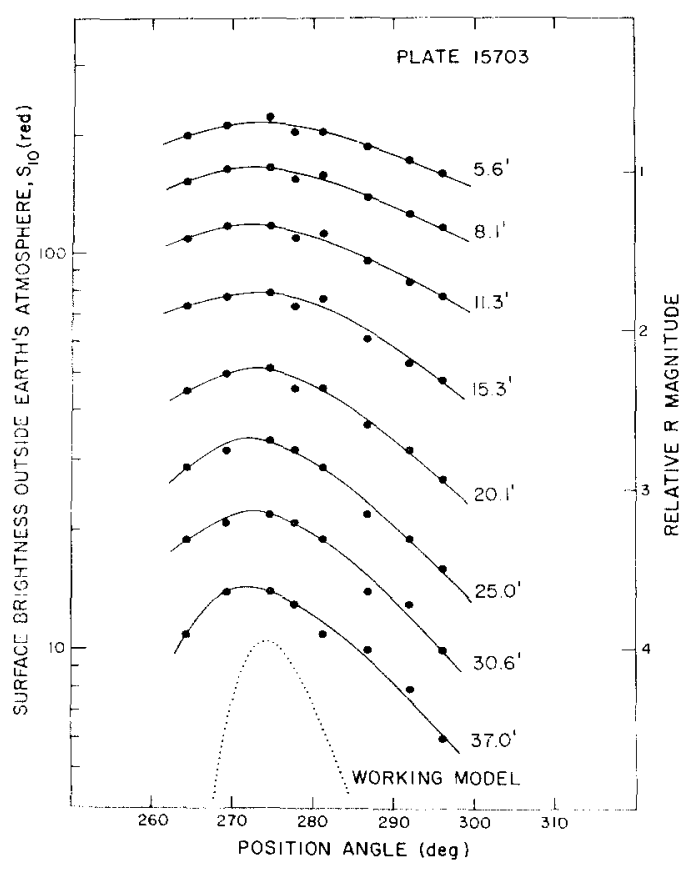

Fic. 7. The distribution, in position angle, of surface brightness in the anti-tail of Comet Kohoutek on plate 15703 at several angular distances from the nucleus. For details, see the caption to Fig. 4.

Paper I by a factor of at least 20 , and it thus appears that the emission rate of the dust should be reduced by a comparable factor. This conclusion would be in line with a suggestion put forward in Paper I that the apparent order-of-magnitude discrepancy between the dust-output rates from Comet Kohoutek on the one hand and from Comets Arend-Roland and Bennett on the other was due to the inaccessibility, at the time of completion of Paper I, of a reliable source for absolute calibration and is therefore not real. A proper account of evaporation, however, will somewhat increase the final estimate for the loss of mass of the dust from the comet.

VII. Preliminary Physical INTERPRETATION OF THE OBSERVED Radial Profiles of the Anti-'Tail

The physical significance of the progressively increasing gradient of the radial

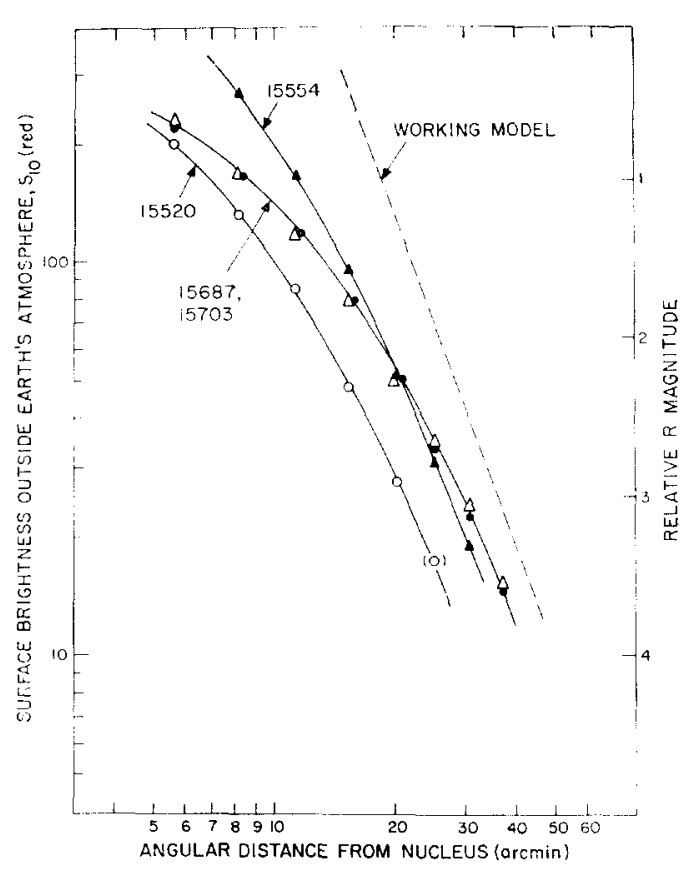

Fici. 8. The maximum surface brightness in the anti-tail of Comet Kohoutek on the four plates as a function of the angular distance from the nucleus. The inverse 2.8 power law predicted by the working model is also plotted.

scans emerges from a model formulated by Finson and Probstein (1968) for small particle-ejection velocities. The model relates the modified surface density of the emitted particles (which is proportional to the surface brightness) to their emission flux and size distribution and to the area they occupy in the plane of the sky. As long as we consider only larger particles, for which the acceleration ratio $1-\mu$ of solar radiation pressure to solar gravity does not exceed $\sim 0.01$, the relative surface brightness in a particular direction away from the nucleus is related to $\mathrm{I}-\mu$ in a simple way. First, the radial direction is practically identical with a synchrone. Second, the projected area occupied by the particles is proportional to $1-\mu$. Third, for a constant particle density, the particle diameter $\delta$ is inversely proportional to $1-\mu$ because the scattering efficiency of the large particles for radiation pressure is practically independent of the particle size. And fourth, the distance $R$ of the 


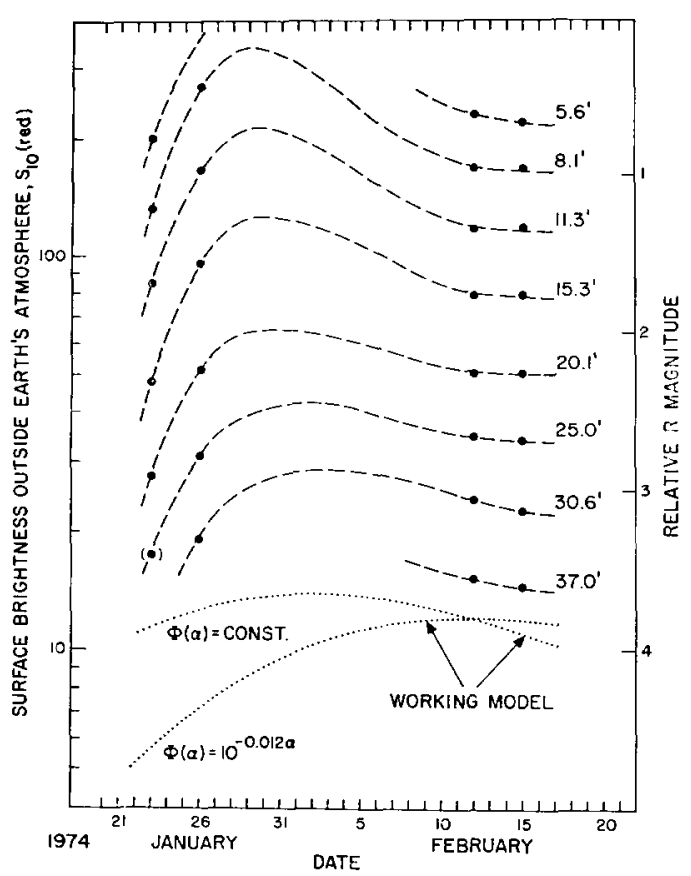

Fia. 9. The maximum surface brightness of the anti-tail of Comet Kohoutek at several angular distances from the nucleus as a function of time. The dashed curves show only one of many possible fits to the observations. For comparison, relative brightness variations in time predicted by the working model are plotted for two idealized cases: the asteroidal-type phase law $\Phi=10^{-0.012 \alpha}$ ( $\alpha$ is the phase angle), and a constant phase effect $\Phi=$ const.

large particles from the nucleus is proportional to $1-\mu$. The relative surface brightness at distance $R$ is then

$$
I(R) \sim f(1-\mu)(1-\mu)^{-1},
$$

where $f(1-\mu)$ is related to the particlesize distribution function $g(\delta)$ as follows:

$$
f(1-\mu) \sim \delta^{4} g(\delta) \sim(1-\mu)^{-4} g(\delta) .
$$

We established in Paper I that there was an excess of heavier particles in the antitail, which we interpreted as possible evidence for a significant loss of radius of the dust particles due to evaporation near the perihelion passage. With a sufficient body of observational information, we can now test this hypothesis more quantitatively.
Consider an initial (preevaporation) distribution function of particle diameters of the form

$$
g(\delta) d \delta \sim \delta^{-u} d \delta
$$

where $u$ is a constant. Consider further that, as a result of intense solar heating over a limited period of time, an outer layer $\Delta \delta / 2$ in thickness is lost by evaporation from each particle. This amount is assumed to be independent of the particle size for material of given structure and composition. A particle of initial diameter $\delta$ now has a smaller diameter, $\gamma=\delta-\Delta \delta$, and the particle-size distribution (3) changes to

$$
g(\gamma) d \gamma \sim(\gamma+\Delta \delta)^{-u} d \gamma .
$$

The logarithmic slope of this distribution,

$$
t(\gamma)=-\partial \log _{e} g(\gamma) / \partial \log _{e} \gamma,
$$

depends on the diameter $\gamma$ and is related to the initial exponent $u$ by

$$
t(\gamma)=u /[1+(\Delta \delta / \gamma)]
$$

Since $\gamma=C(1-\mu)^{-1}$ (where $C$ is virtually a constant for particles of constant density, and $1-\mu$ now refers to $\gamma$ rather than to $\delta$ ), we can write (6) as

$$
1 / t=(1 / u)+(\Delta \delta / C u)(1-\mu) .
$$

On the other hand, we now have $I \sim \gamma^{5} g(\gamma)$, so that the radial brightness gradient is

$$
\begin{aligned}
w=-\frac{\partial \log _{e} I(R)}{\partial \log _{e} R} & =-\frac{\partial \log _{e} I(\gamma)}{\partial \log _{e} \gamma} \cdot \frac{\partial \log _{e} \gamma}{\partial \log _{e} R} \\
& =5-t(\gamma) .
\end{aligned}
$$

Deriving $t(\gamma)$ for a number of angular distances on each of the scanned profiles, assigning the proper value of $1-\mu$ (depending not only on the distance $R$, but also on the time of observation and on the position angle of the scan), and plotting the resulting pairs $1-\mu$ versus $1 / t$, we can test the hypothesis of particle evaporation by comparing such a plot with the linear relation predicted by (7). The plot, based on the four plates and including only the sections of the anti-tail where its relative brightness exceeded $2 \%$ of the sky intensity (to avoid unrealistic gradients from very faint areas), is exhibited in Fig. 10. 


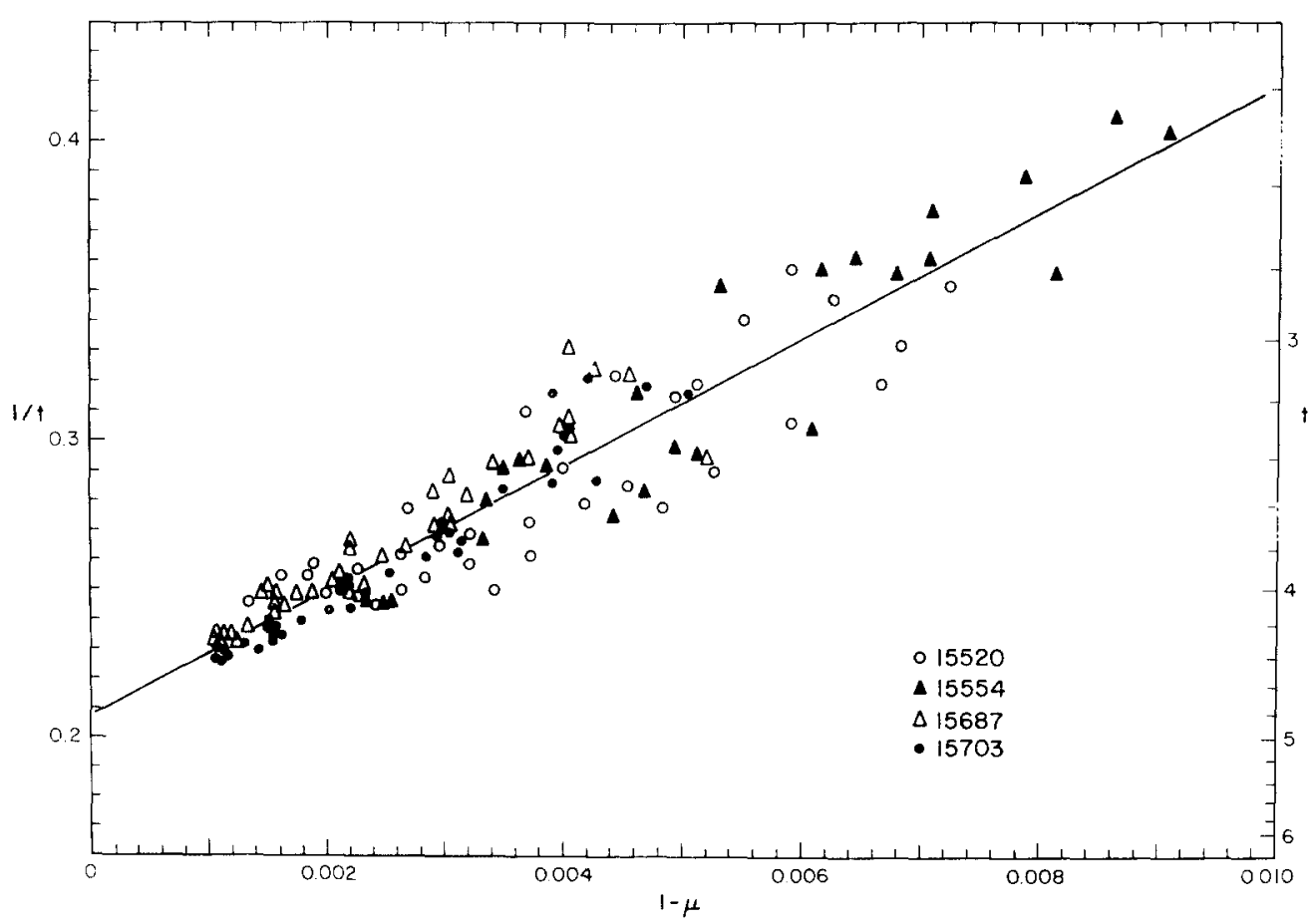

Fic. 10. A plot of particle acceleration $1-\mu$ versus the logarithmic gradient $t$ of the size distribution of the vaporizing particles in the anti-tail.

A least-squares solution indicates a complete absence of nonlinearity (the mean error of the quadratic term came out 4.5 times as large as the term itself) and provides numerical values for two important characteristics of the population of large dust particles in Comet Kohoutek: the exponent $u$ of the initial particle-size distribution (3) and the evaporation loss in the particle diameter $\Delta \delta$. The two quantities derived from both the separate plates and the whole set are listed in Table III.
The table also includes the population index $s$ of the preevaporation differential particle-mass distribution $m^{-s} d m$, which relates to the particle-size exponent $u$ of $(3)$,

$$
s=(u+2) / 3
$$

and the acceleration ratio $(1-\mu)_{\max }$ of the largest particles that evaporated completely (i.e., whose $\delta=\Delta \delta$ ).

Taken at face value, the data of Table III appear to indicate rather consistently that

'TABLL' $11 \mathrm{I}$

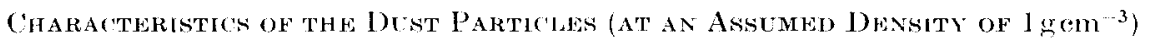

\begin{tabular}{|c|c|c|c|c|}
\hline Plate & $\begin{array}{l}\text { Initial size } \\
\text { distribution } \\
\text { exponent, } u\end{array}$ & $\begin{array}{l}\text { Initial } \\
\text { population } \\
\text { index, s }\end{array}$ & $\begin{array}{l}\text { Evaporation loss in } \\
\text { particle diameter } \Delta \delta \\
(\mu \mathrm{m})\end{array}$ & $\begin{array}{c}\text { Initial repulsive force for } \\
\text { largest completely evaporated } \\
\text { particlos, }(1-\mu)_{\max }\end{array}$ \\
\hline 15520 & $4.72 \pm 0.16$ & $2.24 \pm(0.1) 5$ & $103 \pm 10$ & $0.0113 \pm 0.0011$ \\
\hline 15554 & $5.17 \pm 0.27$ & $2.39+0.09$ & $142 \pm 13$ & $0.0082 \pm 0.0007$ \\
\hline 15687 & $4.79 \pm 0.09$ & $2.26 \pm 0.03$ & $131 \pm 8$ & $0.0089 \pm 0.0005$ \\
\hline 15708 & $5.06 \pm 0.0 \%$ & $2.35 \pm 0.02$ & $147 \pm 6$ & $0.0080 \pm 0.0003$ \\
\hline All & $4.81 \pm 0.06$ & $2.27 \pm 0.02$ & $118 \pm 4$ & $0.0099 \pm 0.0003$ \\
\hline
\end{tabular}


the population index is $s \simeq 2.3$ (and the size-distribution exponent $u \simeq 5$ ) and that only those particles initially larger than $100-150 \mu \mathrm{m}$ in diameter (at an assumed density of $1 \mathrm{~g} \mathrm{~cm}^{-3}$ ) survived the exposure to solar heat. Comparison with Fig. 7 of Paper I suggests that the effective vaporization heat of the anti-tail particles a mounts to about $46 \mathrm{kcal} \mathrm{mole}^{-1}$. However, an uncertainty is involved in these results, owing to the fact that particle evaporation also affects the particle's future dynamics because it implies a change in the magnitude of the radiation pressure. Therefore, the present interpretation is only preliminary, and the derived loss rate of particle radius due to evaporation, as well as the actual emission rate of the dust, is still subject to further analysis. The improved model of the anti-tail will have to account for the dynamical effect of particle evaporation (variable $1-\mu$ ).

\section{Appendix A}

\section{Effects of Differential Refraction on}

Distance and Direction in the Anti-Tail at Low Altitudes

Because of the sharp increase in the refraction correction of the spherical coordinates at low altitudes, it is desirable to establish the effects of refraction on the distance and directional scales in the anti-tail.

Let $\alpha_{1}, \delta_{1}$ be the refraction-free coordinates of the comet's nucleus; $\alpha_{2}, \delta_{2}$, those of a point in the anti-tail; and, similarly, $\alpha_{1}^{\prime}, \delta_{1}^{\prime}, \alpha_{2}^{\prime}, \delta_{2}^{\prime}$, the corresponding coordinates affected by refraction. The corrections for refraction are, therefore, $\Delta \alpha_{i}=\alpha_{i}-\alpha_{i}^{\prime}, \Delta \delta_{i}=\delta_{i}-\delta_{i}^{\prime}(i=1,2)$. Since we consider only small arcs, then for the refraction-free angular distance $\Delta$ of the point $\left(\alpha_{2}, \delta_{2}\right)$ from the nucleus, we can write

$$
\begin{aligned}
\Delta^{2}=\left[\left(\alpha_{2}^{\prime}+\Delta \alpha_{2}\right)-\left(\alpha_{1}^{\prime}+\Delta \alpha_{1}\right)\right]^{2} \\
\quad \times \cos ^{2} \frac{1}{2}\left[\left(\delta_{1}^{\prime}+\Delta \delta_{1}\right)+\left(\delta_{2}^{\prime}+\Delta \delta_{2}\right)\right] \\
\quad+\left[\left(\delta_{2}^{\prime}+\Delta \delta_{2}\right)-\left(\delta_{1}^{\prime}+\Delta \delta_{1}\right)\right]^{2} .
\end{aligned}
$$

Denoting the refraction-affected distance of point $\left(\alpha_{2}, \delta_{2}\right)$ as $\Delta^{\prime}$ and realizing that

$$
\begin{aligned}
& \cos \frac{1}{2}\left[\left(\delta_{1}^{\prime}+\Delta \delta_{1}\right)+\left(\delta_{2}^{\prime}+\Delta \delta_{2}\right)\right] \\
& \approx \cos \frac{1}{2}\left(\delta_{1}^{\prime}+\delta_{2}^{\prime}\right) \approx \cos \delta_{1}^{\prime}, \\
& \left(\alpha_{2}^{\prime}-\alpha_{1}^{\prime}\right)^{2} \cos ^{2} \frac{1}{2}\left(\delta_{1}^{\prime}+\delta_{2}^{\prime}\right) \\
& +\left(\delta_{2}^{\prime}-\delta_{1}^{\prime}\right)^{2}=\Delta^{\prime 2}, \\
& \left|\Delta \alpha_{2}-\Delta \alpha_{1}\right| \leqslant\left|\alpha_{2}^{\prime}-\alpha_{1}^{\prime}\right| \text {, } \\
& \left|\Delta \delta_{2}-\Delta \delta_{1}\right| \ll\left|\delta_{2}^{\prime}-\delta_{1}^{\prime}\right| \text {, } \\
& \Delta^{2}-\Delta^{\prime 2} \approx 2 \Delta^{\prime}\left(\Delta-\Delta^{\prime}\right) \text {, }
\end{aligned}
$$

we find that the refraction correction in distance amounts to

$$
\begin{gathered}
\Delta-\Delta^{\prime} \doteq\left(1 / \Delta^{\prime}\right)\left[\left(\alpha_{2}^{\prime}-\alpha_{1}^{\prime}\right)\left(\Delta \alpha_{2}-\Delta \alpha_{1}\right)\right. \\
\left.\times \cos ^{2} \delta_{1}^{\prime}+\left(\delta_{2}^{\prime}-\delta_{1}^{\prime}\right)\left(\Delta \delta_{2}-\Delta \delta_{1}\right)\right]
\end{gathered}
$$

Similarly, the refraction-free position angle $p$ of the point $\left(\alpha_{2}, \delta_{2}\right)$ relative to the nucleus is

$$
\begin{aligned}
\tan p= & \frac{\left(\alpha_{2}^{\prime}+\Delta \alpha_{2}\right)-\left(\alpha_{1}^{\prime}+\Delta \alpha_{1}\right)}{\left(\delta_{2}^{\prime}+\Delta \delta_{2}\right)-\left(\delta_{1}^{\prime}+\Delta \delta_{1}\right)} \\
& \times \cos \frac{1}{2}\left[\left(\delta_{1}^{\prime}+\Delta \delta_{1}\right)+\left(\delta_{2}^{\prime}+\Delta \delta_{2}\right)\right] .
\end{aligned}
$$

With the approximations (A2), the relation between the refraction-free and the refraction-affected position angles $p$ and $p^{\prime}$ is expressed as

$$
\begin{aligned}
\tan p \doteq & \tan p^{\prime} \\
& \times\left(1+\frac{\Delta \alpha_{2}-\Delta \alpha_{1}}{\alpha_{2}^{\prime}-\alpha_{1}^{\prime}}-\frac{\Delta \delta_{2}-\Delta \delta_{1}}{\delta_{2}^{\prime}-\delta_{1}^{\prime}}\right),
\end{aligned}
$$

and, with $p$ and $p^{\prime}$ in degrees, the refraction correction in direction becomes

$$
\begin{array}{rl}
p-p^{\prime}=2 & 2.65 \sin 2 p^{\prime} \\
& \times\left(\frac{\Delta \alpha_{2}-\Delta \alpha_{1}}{\alpha_{2}^{\prime}-\alpha_{1}^{\prime}}-\frac{\Delta \delta_{2}-\Delta \delta_{1}}{\delta_{2}^{\prime}-\delta_{1}^{\prime}}\right) .
\end{array}
$$

\section{Appendix B}

\section{Calibration of the Ambient Sky Brightness from the Trail of a Star of Known Magnitude}

We assume that a star's image on a calibrated plate, guided on a moving object (comet), appears in the form of a trail of length $L$ and characteristic width $W$ (arcsec). We further assume that a photometric scan across a section of the 
trail is available (such as the one in Fig. 3) and that the diaphragm used is a slit of length $l$ and width $w$ (arcsec), where $l \leqslant L$ and $w \ll W$. Converting the photographic densities across the measured profile of the star to relative intensities and fixing the level of the sky brightness $I_{\mathrm{S}}$ on the scan, we wish to establish the ratio of the brightness of the scanned section of the star to that of the ambient sky. It appears that the excess relative intensity $I$ across the star's profile can be fitted by a probability-type curve,

$$
I(x)=I_{*} \exp \left[-\left(x-x_{0}\right)^{2} / 2 \sigma^{2}\right],
$$

where $x$ (arcsec) is read from an arbitrary origin $x_{0}, I_{*}$ is the peak relative brightness at the center of the star's profile, and $\sigma$ is the width factor (aresec). Establishing $I(x)$ for a number of $x$ from the scan, we can determine $I_{*}$ and $\sigma$ by least squares. Equation (Bl) can be written as

where

$$
\log _{e} I(x)=A+B x+C x^{2}
$$

$$
\begin{aligned}
& A=\log _{e} I_{*}-\left(x_{0}^{2} / 2 \sigma^{2}\right), \\
& B=x_{0} / \sigma^{2}, \\
& C=-1 / 2 \sigma^{2},
\end{aligned}
$$

so that

$$
l_{*}=\exp \left[A-\left(B^{2} / 4 C\right)\right]
$$

and

$$
\sigma=(-20)^{-1 / 2}
$$

The integrated intensity in the scanned section of the star's trail is

$$
\int_{-\infty}^{\infty}(I / w) d x=(2 \pi)^{1 / 2} I_{*} \sigma / u \text {, }
$$

and the brightness contribution of the whole trail of the star is related to the star's magnitude $H_{*}$ :

$$
(2 \pi)^{1 / 2} I_{*}(\sigma / u)(L / l)=r \times 10^{-0.4 H_{*}},
$$

where $c$ is a constant. The surface brightness of the ambient sky $H_{\mathrm{s}}$ (magnitude per square arcsec) can similarly be expressed through the relative brightness $I_{\mathrm{S}}$ and the slit dimensions

$$
I_{\mathrm{S}} / l w=c \times 10^{-0.4 \mathrm{H}_{\mathrm{S}}} .
$$

The elimination of $c$ from (B7) and (B8) then immediately gives the result

$$
H_{\mathrm{S}}=H_{*}+2.5 \log _{10}\left[(2 \pi)^{1 / 2} I_{*} \sigma L / I_{\mathrm{S}}\right],
$$

or, in terms of the equivalent number of stars of magnitude 10 per square degree,

$$
\begin{aligned}
S_{10} & =1.296 \times 10^{11-0.4 H_{\mathrm{s}}} \\
& =\frac{5.170 I_{\mathrm{S}}}{I_{*} \sigma L} 10^{10-0.4 H_{*}} .
\end{aligned}
$$

\section{Achnowledgments}

We thank Professor $W$. Liller, Center for Astrophysics, for the determination of red magnitudes of the calibration stars. We also thank Messrs. C. Miller and J. Conlon, both of PhotoMetries, Ine., Lexington. Massachusetts, for the high-quality photometric tracings. The plates were secured as part of a project supported by grant (XP-37486 from the National Science Foundation. All work on the plates was supported by wrant NGR 09-015-159 from the National Aeronautics and Space Administration. Support from the National Science Foundation grant MPS $75-(01421$ is also acknowledged.

\section{References}

Fixson, M. L., AND Probstein, R. F. (1968). A theory of dust comets. 1. Model and equations. Astrophys. J. 154, 327-352.

LRIARTE, B. (1967). UBV photoelectric photometry for faint stars in the Pleiades cluster. Bol. Obs. Tonantzintla y Tacubaya 4, 79 83.

Iriarte, B.. Johnson, H. L., Mitchell, R. I., AND WISNIEWSKI. W. K. (1965). Five-color photometry of bright stars. $\$ k y$ d $T$ Tel. $\mathbf{3 0}$, 2131.

JамвоR, B. I. (1973). The split tail of Comet Seki-Lines. Astrophys. J. 185, 727 - 734.

Johnson, H. L., AND Mitcheld, R. I. (1962). A completely digitized multi-color photometer. Comm. Lunar Planet. Lab. 1, 73 .81.

Marsdex, B. G. (1974). Comet Kohoutek (1973f). TALC No. 2684.

MildFr, F. D. (1958). Filters for comet photography Comet Mrkos, 1957d. Publ. Astrom. Soc. P'acific 70, 279-284.

Millfer, F. D. (1962). The type I tail of comet 1955e. Publ. Astron. Soc. Pacific 74, 60-65.

Millek. F. D. (1967). Photometry of the $C_{2}$ coma of Comet Burnham (1960 II). Astron. .J. $72,487-492$.

SEkANINA, $Z$. (1974). On the nature of the ant $i-$ tail of Comet Kohoutek 1973f. I. A working model. Icarus 23, 502-518. (Papłr I.)

Sheanina, Z., ANd Miller, F. D. (1973). (omet Bennett 1970 II. Science 179, 565-567. 